\title{
Research Article \\ Combining Low-Level Features for Semantic Extraction in Image Retrieval
}

\author{
Q.Zhang and E. Izquierdo \\ Multimedia and Vision Laboratory, Electronic Engineering Department, Queen Mary University of London, London E14NS, UK
}

Received 9 September 2006; Revised 28 February 2007; Accepted 16 April 2007

Recommended by Hyoung Joong Kim

\begin{abstract}
An object-oriented approach for semantic-based image retrieval is presented. The goal is to identify key patterns of specific objects in the training data and to use them as object signature. Two important aspects of semantic-based image retrieval are considered: retrieval of images containing a given semantic concept and fusion of different low-level features. The proposed approach splits the image into elementary image blocks to obtain block regions close in shape to the objects of interest. A multiobjective optimization technique is used to find a suitable multidescriptor space in which several low-level image primitives can be fused. The visual primitives are combined according to a concept-specific metric, which is learned from representative blocks or training data. The optimal linear combination of single descriptor metrics is estimated by applying the Pareto archived evolution strategy. An empirical assessment of the proposed technique was conducted to validate its performance with natural images.
\end{abstract}

Copyright (C) 2007 Q. Zhang and E. Izquierdo. This is an open access article distributed under the Creative Commons Attribution License, which permits unrestricted use, distribution, and reproduction in any medium, provided the original work is properly cited.

\section{INTRODUCTION}

The problem of retrieving and recognizing patterns in images has been investigated for several decades by the image processing and computer vision research communities. Learning approaches, such as neural networks, kernel machines, statistical, and probabilistic classifiers, can be trained to obtain satisfactory results for very specific applications [1-3]. Unfortunately, fully automatic image recognition using highlevel semantic concepts is still an unfeasible task. Though low-level feature extraction algorithms are well understood and able to capture subtle differences between colors, statistic and deterministic textures, global color layouts, dominant color distributions, and so forth, the link between such lowlevel primitives and high-level semantic concepts remains an open problem. This problem is referred to as "the semantic gap." To narrow this gap is a challenge that has captured the attention of researchers in computer vision, pattern recognition, image processing, and other related fields, evidencing the difficulty and importance of such technology and the fact that the problem remains unsolved $[4,5]$.

In this paper, an object-oriented approach for semanticbased image retrieval is presented. Two important aspects of semantic-based image annotation and retrieval are considered: retrieval of images containing a given semantic con- cept and fusion of different low-level features. The first aspect relates to the fact that in most cases users are interested in finding single objects rather than whole scene depicted in an image. Indeed, when watching images, human beings tend to look for single semantical meaningful objects and unconsciously filter out surrounding elements and other objects in complex scenes. The second aspect, that is, the joint exploitation of different low-level image descriptions is motivated by the fact that single low-level descriptors are not suitable for interpreting human understanding into visual description by machines. Combining them in a concept-specific manner may help to solve the problem, but different visual features and their similarity measures are not designed to be combined naturally in a meaningful way. Thus, questions related to the definition of a metric joining several similarity functions require careful consideration. The low-level descriptors used in this work are based on specific and different visual cues representing various aspects of the content. The aim is to learn associations between complex combinations of low-level descriptions and semantic concepts. It is expected that low-level visual primitives complement each other and jointly build a multidescriptor that can represent the underlying visual context in a semantic way.

A large number of different features can be used to obtain content representations that could potentially capture 
or describe semantic objects in images. The difficulty of the problem arises from the different nature of the features used as described in $[6,7]$. Different features are extracted using different algorithms and the corresponding descriptors have individually specific syntaxes. The unavoidable effect is that different descriptors "live" in different feature spaces with their own metrics and statistical behavior. As a consequence, they cannot be naturally mixed to convey semantic meanings. Therefore, finding the right mixture of low-level features and their corresponding metrics is important to bridge the semantic gap. The idea of combining descriptors and their metrics in an effort to represent semantic concepts has been addressed for years in pattern recognition. In [8], semantic objects in images were represented by weighted lowlevel features. Weights were derived from standard deviation over relevant examples. In [9], a similar approach with combination of query point movement and weight update was reported. Alternative many computer vision approaches were based on local interest point detectors and descriptors invariant to geometric and illumination variations [10]. In [11], two combination mechanisms for MPEG-7 visual descriptors were proposed: multiple feature direct combination and multiple feature cascaded combination. They aimed at combining the output of five different expert classifiers trained using three different low-level features. In the system introduced in [12], several low-level image primitives were combined in a suitable multiple feature space modeled in a structured way. SVMs with an adaptive convolution kernel were used to learn the structured multifeature space. However, this approach suffers from an "averaging" effect in the structure construction process so that no much reward is added to the performance.

Contrasting these and other approaches from the literature, in this paper an object-oriented image retrieval approach based on image blocks is presented. The approach is designed to exploit underlying low-level properties of elementary image blocks that constitute objects of interest. Images are divided into small blocks with potentially variable sizes. The goal is to reduce the influence of noise coming from the background and surrounding objects, in order to identify a suitable mixture of low-level patterns that best represent a given semantic object in an image. The approach employs a multiobjective optimization (MOO) technique to find an optimal metric combining several low-level image primitives in a suitable multidescriptor space [13]. Visual primitives are combined according to a concept-specific metric, which is "learned" from some representative blocks. The optimal linear combination of single metrics is estimated by applying multiobjective optimization based on a Pareto archived evolution strategy (PAES) [14]. The final goal is to identify key patterns common to all of the data samples representing an average signature for the object of interest.

The paper is organized as follows. An overview of the proposed approach and an outline of the framework are given in Section 2. The proposed multiobjective optimization approach for image retrieval and classification along with related background introductions are presented in Section 3. Selected experiment results from a very comprehensive empirical study for evaluation are reported in
Section 4. The paper closes with conclusions and future work in Section 5.

\section{AN OBJECT-BASED FRAMEWORK FOR IMAGE RETRIEVAL}

In most image retrieval scenarios, users' attention focuses on single objects. For that reason, in this work the emphasis is on single objects rather than on the whole scene depicted in the image. However, segmentation is not assumed, since we argue that segmenting an image into single semantically meaningful objects is almost as challenging as the semantic gap problem itself. To deal with objects, a very simple approach is taken based on small image blocks of regular size called elementary building blocks of images. The proposed technique was inspired by three simple observations: users are mostly interested in finding objects in images and do not care about the surroundings in picture; elementary building elements are closer to low-level descriptions than whole scenes; objects are made up of elementary building elements. In Figure 1, an example is presented illustrating these observations. The highlighted elementary blocks are clearly representatives of the concepts "tiger," "vegetation," and "stone." These blocks are small enough to be contained in a single object and large enough to convey information about the underlying semantic object.

The proposed framework for object-based semantic image retrieval is outlined in Figure 2 and consists of three main processing stages: preprocessing, multidescriptor metric estimation, and retrieval or classification.

\subsection{Preprocessing}

The preprocessing stage, as depicted in the left-side module, is conducted offline and consists of four different steps. Firstly, each image in the database is partitioned into a fixed grid of $x \times y$ blocks. The size of the grid is chosen adaptively according to the database to reduce the effect of scaling in images of different sizes. Secondly, low-level features are extracted automatically. Any set of low-level descriptors and features can be used and combined in the proposed approach. In particular, seven visual primitives are used in this paper to assess the performance of the proposed approach: color layout (CLD), color structure (CSD), dominant color (DCD), edge histogram (EHD), texture feature based on Gabor filters (TGF), grey level cooccurrence matrix (GLC), and hue-saturation- value (HSV). Observe that the first four are MPEG-7 descriptors [6], while the other three are wellestablished descriptors from the literature [15-17]. In the third step, given a semantic concept, a set of representative block samples are selected for training by professional users. Here, the semantic concept or object is represented by a given key word, for example, "tiger," and the key word is linked with the representative block set. It is assumed that this representative set conveys the most important information on the objects of concern. Besides, it is required that the representative group encapsulates enough discriminating power to filter the actual relevant blocks to the concept from noise in unrelated blocks. Therefore, in this work, two classes of 


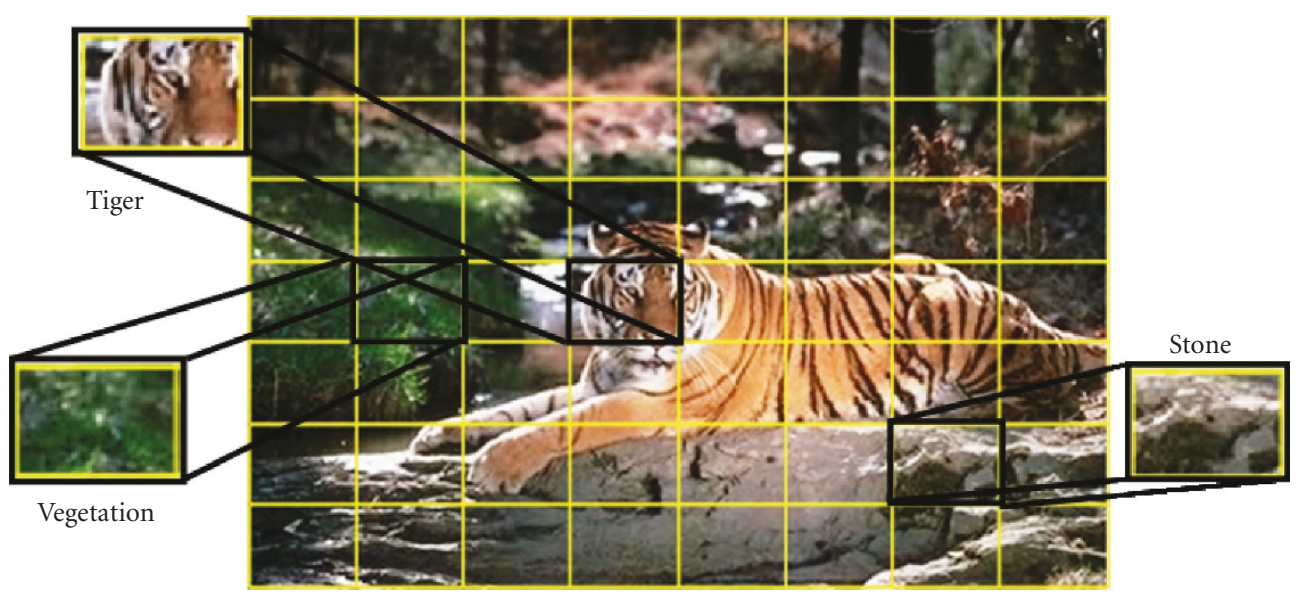

FIGURE 1: Elementary building blocks in an image.

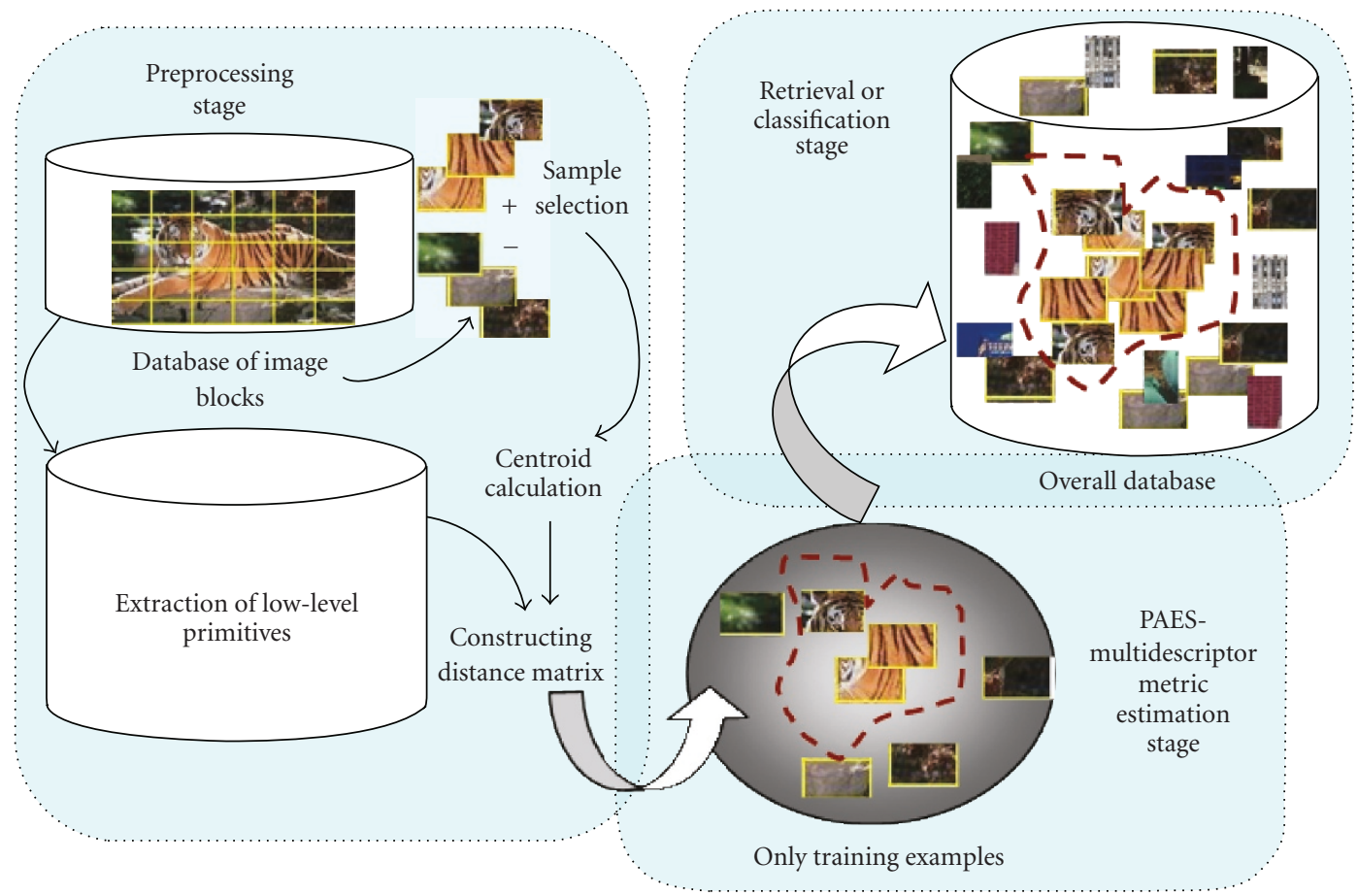

FIgURE 2: Framework overview.

representative samples are selected. The first class contains the most relevant samples for the semantic concept. They are referred to as "positive samples." The second class contains "negative samples" which are irrelevant and have little in common with the semantic concept. The combination of both positive and negative samples builds the training set. Once the training set is available, finally a centrod is calculated for the positive training sets using each one of the corresponding similarity measures of the considered feature spaces. Thus, for $L$ feature spaces, a total of $L$ centroids are calculated. The training set and its centroids are then used for building a distance matrix for the optimization strategy that will be further described in Section 3.

\subsection{Multidescriptor metric estimation and retrieval stages}

After preprocessing, the underlying visual pattern of semantic concepts in the multifeature space are learned using the selected training set. multiobjective optimization is used to find a suitable metric in multifeature space. Specifically, the Pareto archived evolution strategy is adopted to solve the underlying optimization problem. The final stage is the actual retrieval. Here, block-based retrieval using an optimized metric in multidescriptor space is performed. These two processing steps build the backbone of the proposed approach and are elaborated in the next two sections. 


\section{A SIMILARITY MEASURE FOR IMAGE RETRIEVAL USING MULTIOBJECTIVE OPTIMIZATION}

In natural images, semantic concepts are complex and can be better described using a mixture of single descriptors. However, low-level visual descriptors have nonlinear behaviors and their direct combination may easily become meaningless. Among an infinite number of potential ways to combine similarity functions from different feature spaces, the most straightforward candidate for a distance measure in multifeature space is a linear combination of the distances defined for single descriptors. Even in this case, it is difficult to estimate the levels of importance for each feature in the underlying linear combination. The work described in this paper focuses on obtaining an "optimal" metrics based on a linear combination of single metrics of descriptors in a multifeature space. It is an expanded work based on the authors' previous paper [18]. To harmonize a diversity of characteristics in low-level visual descriptors, a strategy similar to multiple decision making is proposed. This kind of strategies aims at optimizing multiple objectives simultaneously [13]. The challenge here is to find suitable weights for combining several descriptor metrics.

\subsection{Build up the multifeature distance matrix}

Let $\mathbf{B}=\left\{b^{(k)} \mid k=1, \ldots, K\right\}$ be the training set of elementary building blocks selected in the preprocessing stage. Here, $K$ is the number of training blocks. B is directly linked to a given semantic concept or key word. Clearly, for each new semantic concept a new training set needs to be selected by an expert user or annotator. For each low-level descriptor, a centroid is calculated in $\mathbf{B}$ by finding the block with the minimal sum of distances to all other blocks in $\mathbf{B}$. That is, if $\bar{v}_{l}$ represents the centroid of the set for a particular feature space $l$, then $\overline{\mathbf{V}}=\left\{\bar{v}_{1}, \bar{v}_{2}, \ldots, \bar{v}_{L}\right\}$ denotes a virtual overall centroid across different features of a particular training set $\mathbf{B}$. Here, $L$ is the number of low-level features or feature spaces considered. $\overline{\mathbf{V}}$ is referred to as a "virtual" centroid since in general, it does not necessarily represent a specific block of $\mathbf{B}$. Depending on the used feature, each centroid may be represented by a different block in $\mathbf{B}$. Taking $\overline{\mathbf{V}}$ as an anchor, the following set of distances can be estimated:

$$
d_{l}^{(k)}=d\left(\overline{v_{l}}, v_{l}^{(k)}\right), \quad k=1, \ldots, K, l=1, \ldots, L,
$$

where $v_{l}^{(k)}$ denotes the $l$ th feature vector of the $k$ th block of the training set, $\mathbf{V}^{(k)}$ is the feature vector set of the $k$ th block and $d_{l}^{(k)}$ is the similarity measure for the $l$ th feature space. Using (1), a $K \times L$-matrix of distance values is then generated. For a given key word or semantic concept representing an object and its corresponding training set $\mathbf{B}$, the following matrix is built:

$$
\begin{array}{cccc}
d_{1}^{(1)} & d_{2}^{(1)} & \cdots & d_{L}^{(1)} \\
d_{1}^{(2)} & d_{2}^{(2)} & & d_{L}^{(2)} \\
\vdots & & \ddots & \vdots \\
d_{1}^{(K)} & d_{2}^{(K)} & \cdots & d_{L}^{(K)}
\end{array}
$$

In (2), each row contains distances of different features of the same block, while each column displays distances of a feature for different blocks. The distance matrix (2) is the basis which the objective functions for optimization are built from.

\subsection{The need for multiobjective optimization}

Let $D: \mathbf{V} \times \overline{\mathbf{V}} \rightarrow \mathfrak{R}$ be the distance between a set of feature vectors $\mathbf{V}$ and the virtual centroid $\overline{\mathbf{V}}$ in the underlying multifeature space. As mentioned before, the most straightforward candidate for the combined metric $D$ in a multifeature space for an image block is the weighted linear combination of the feature-specific distances:

$$
D^{(k)}\left(\mathbf{V}^{(k)}, \overline{\mathbf{V}}, \mathbf{A}\right)=\sum_{l=1}^{L} \alpha_{l} d_{l}^{(k)}\left(\bar{v}_{l}, v_{l}^{(k)}\right),
$$

where $d_{l}^{(k)}$ is the distance function as defined in (1) and $\mathbf{A}=\left\{\alpha_{l}, l=1, \ldots, L\right\}$ is the set of weighting coefficients we are seeking to optimize. Each row in (2) is reformed into an objective function such as in (3). According to (3), now the problem consists of finding the optimal set $\mathbf{A}$ of weighting factors $\alpha$, where optimality is regarded in the sense of both concept representation and discrimination power. The underlying argument here is that semantic objects can be more accurately described by a suitable mixture of low-level descriptors than by single ones. However, this leads to the difficult question about how descriptors can be mixed and what is the "optimal" contribution of each feature. A simple approach to optimize the weighting factors $\alpha$ according to (3) would consider the following combinative objective function:

$$
D(\mathbf{V}, \overline{\mathbf{V}}, \mathbf{A})=\sum_{k=1}^{K} \sum_{l=1}^{L} \alpha_{l} d_{l}^{(k)}\left(\bar{v}_{l}, v_{l}^{(k)}\right), \quad \text { subject to } \sum_{l=1}^{L} \alpha_{l}=1 .
$$

Unfortunately, an approach based on the optimization of (4) leads to unacceptable results due to the complex nature of semantic objects. Semantically, similar objects usually have very dissimilar visual descriptions in some spaces. Even worse, different low-level visual features extracted from the same object class may contradict each other. Consequently, two main aspects need careful consideration when a solution for (3) is sought: firstly, single optimization may lead to biased results; secondly, the contradictory nature of low-level descriptors should be considered in the optimization process. For the sake of clarity, let us consider two simple examples to illustrate these two aspects.

In the first example, the two groups of image blocks shown in Figure 3 are considered. The first group contains 16 blocks of letters with uniform yellow color and blue background but featuring a diversity of edges. It is called the "Letter" group. The second group contains 16 blocks with clear horizontal edges and a diversity of colors. It is called the "Hori" group. In this small experiment, two low-level features are combined according to (4): color layout (CLD) and edge histogram descriptors (EHD). That is, $L=2$ in this specific case. Clearly, the CLD distances between blocks of 


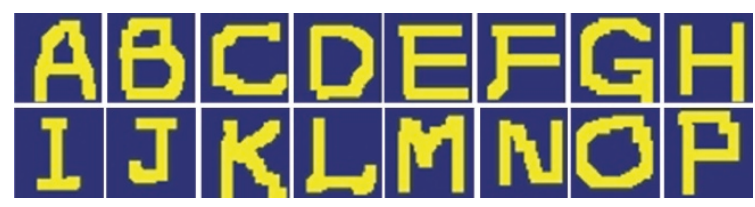

(a)

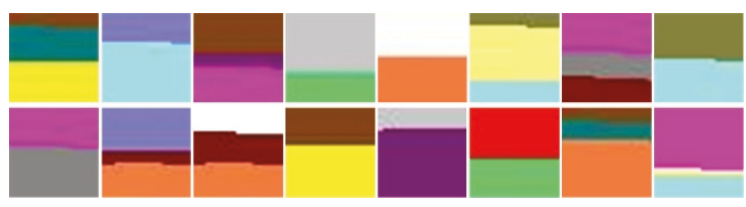

(b)

FIgURE 3: 16 examples of image blocks. "letter" group (a) and "hori” group (b).

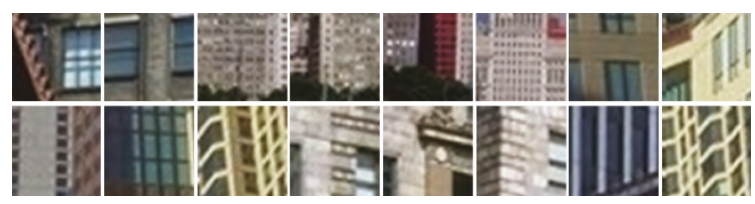

(a)

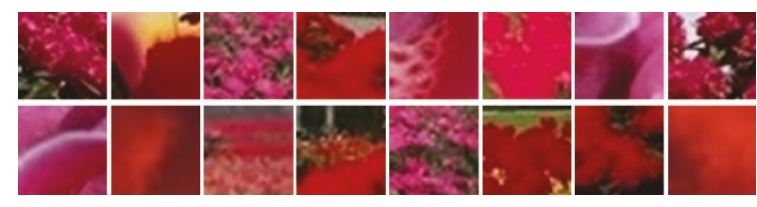

(b)

FIGURE 4: Examples of image blocks for "building" group (a) and "flower" group (b).

the "letter" group are small, while the EHD distances are large. Optimizing (4) leads to the "Boolean" weights 1 for CLD and 0 for EHD. The same process applied to the "hori" group leads to the "boolean" weights 0 for CLD and 1 for EHD. Actually, it is straightforward to prove that the optimization of (4) always results in a "boolean" decision in which a single feature get assigned the weight 1 and all the others the weight 0 . Basically, the reason behind is this simple approach leads to a "winner takes all" result in which the potential contribution of other features to the description of a semantic object is completely ignored. Here, the winner is always the low-level feature with the smallest sum of distances over all training blocks.

The second example aims at illustrating the conflicting nature of descriptors. Here, the blocks illustrated in Figure 4 are considered. The first group consists of 16 blocks selected from images containing buildings and it is called the "building" group. The second group consists of 16 blocks containing red flowers and it is called the "flower" group.

Considering the "flower" group and its intrinsic semantic concept (flower), a color descriptor will identify blocks in which the red color is dominant. The dominance of color over other descriptors, such as edge or texture, is less significant than the dominance of color in the "letter" group in Figure 3. Actually, in this case, the edges and textures of the flowers also contribute to the semantic concept "flower." On the other hand, in the "building" group, texture and edges are dominant while color plays a secondary role. That is, the amount of "edgeness" in the flowers is critical to discriminate a red building from a red flower. In either case increasing the "colorness" or the "edgeness" too much will lead to wrong results. The underlying conflict between descriptors discrimination power cannot be solved if a single objective function is considered. An optimal trade-off is needed. Therefore, the optimization model needs to be based on the contribution of each single primitive to the description of the semantic object. Clearly, optimizing a set of potentially contradicting objective functions does not lead to optimum solutions for all objective functions. This is the part where multiple decision making plays an important role. The interaction between different objectives leads to a set of compromised so- lutions, largely known as the Pareto-optimal solutions [13]. Since none of these Pareto-optimal solutions can be declared to be better than others without any further consideration, the initial goal is to find a collection of Pareto-optimal solutions. Once the Pareto-optimal solutions are available, a second processing step is required in which higher-level decision making is performed to choose a single solution among the available solutions. In this paper, PAES is adopted to optimize the metrics combining the visual descriptors. In the second step, the high-level decision making is achieved by selecting the solution for which the sum of all objective solutions is minimal as the final optimal solution. The rationale behind this decision making strategy is that small sums of weighted distances lead to better gathering of all training sample vectors in feature space, which is the target of the overall optimization approach.

\subsection{Multifeature metric optimization}

To ensure a minimum comparability requirement, all the $L$ distances $d_{l}$ are normalized using simple min-max normalization. This transforms the distance output into the range $[0,1]$ by applying

$$
d_{l(\text { new })}=\frac{\left(d_{l}-C\right)}{(D-C)}, \quad l=1, \ldots, L,
$$

where $C$ and $D$ are respectively the minimum and maximum distances between all blocks in the learning set and the corresponding centroid.

Given a semantic object or corresponding key word, the distance matrix (2) is built. The optimization of (3) is then performed by applying PAES on B. Accordingly, the set of objective functions $\mathbf{M}(\mathbf{A})$ is defined as follows:

$$
\mathbf{M}(\mathbf{A})=\left\{\begin{array}{c}
D^{(1)}\left(\mathbf{V}^{(1)}, \overline{\mathbf{V}}, \mathbf{A}\right) \\
D^{(2)}\left(\mathbf{V}^{(2)}, \overline{\mathbf{V}}, \mathbf{A}\right) \\
\cdots \\
D^{(K)}\left(\mathbf{V}^{(K)}, \overline{\mathbf{V}}, \mathbf{A}\right)
\end{array}\right\},
$$

where $\mathbf{A}$ is the collection of decision variables (weighting values), and $D^{(k)}$ is the distance function of the $k$ th block as 

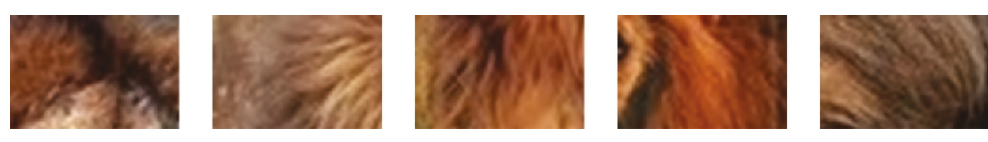

(a)
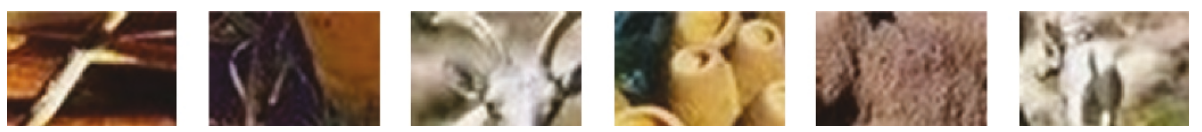

(b)

Figure 5: Positive (a) and negative (b) representative sample blocks of lion. defined in (3). The goal is to find the best set of coefficients $\mathbf{A}=\left\{\alpha_{l} \mid l=1, \ldots, L\right\}$ subject to the following constraint:

$$
\sum_{l=1}^{K} \alpha_{l}=1
$$

The task at hand boils down to minimizing the objective functions (6) generated for all the positive training samples while maximizing the objective functions (6) generated for all the negative training samples. In both cases, simultaneous minimization and maximization are conducted subject to the constraint (7). For the sake of illustration, Figure 5 shows some examples of positive and negative representative blocks for the concept lion.

Observe that, in practice, the virtual centroid $\overline{\mathbf{V}}$ is calculated for the positive samples only. As mentioned before, instead of a single solution, a set of Pareto-optimal solutions is obtained for the positive and negative samples. Using these sets of Pareto-optimal solutions, a final unique solution $\mathbf{A}^{*}$ is estimated in a second "decision making" step. This estimation of $\mathbf{A}^{*}$ can be achieved by minimizing the overall sum of distances between all positive examples and the centroid, while maximizing (spreading) the overall sum of distances between all negative examples and the centroid. In other words, the goal is to minimize the ratio between the overall sum of distances from all positive examples to the centroid and the overall sum of distances from all negative examples to the centroid. Thus, $\mathbf{A}^{*}$ is the set of parameters minimizing:

$$
\min \frac{\sum_{k=1}^{K} D_{+}^{(k)}\left(\mathbf{V}^{(k)}, \overline{\mathbf{V}}, \mathbf{A}_{s}\right)}{\sum_{k=1}^{K} D_{-}^{(k)}\left(\mathbf{V}^{(k)}, \overline{\mathbf{V}}, \mathbf{A}_{s}\right)}, \quad s=1,2, \ldots, S,
$$

where $D_{+}^{(k)}$ and $D_{-}^{(k)}$ represent the distances over positive and negative samples, respectively, $\mathbf{A}_{s}$ is the sth solution in the set of Pareto-optimal solutions and $S$ is the cardinality of the set of Pareto-optimal solutions estimated in the first optimization step.

\subsection{Multiple objective optimization and Pareto archived evolution}

Multiobjective optimization is defined as the problem of finding a vector of decision variables which satisfies given constraints and optimizes a vector of objective functions. These functions form a mathematical description of performance criteria which are usually in conflict with each other.
Hence, the term "optimizes" means finding a solution which gives good or acceptable values for all the objective functions. It can be mathematically stated as finding a particular vector of decision variables $\mathbf{A}^{*}=\left\{\alpha_{1}^{*}, \alpha_{2}^{*}, \ldots, \alpha_{L}^{*}\right\}^{T}$ satisfying $P$ constraints $g_{p}(\mathbf{A}) \geq 0, p=1,2, \ldots, P$ and at the same time optimizing the set of vector functions $\mathbf{M}(\mathbf{A})=$ $\left\{D_{1}(\mathbf{A}), D_{2}(\mathbf{A}), \ldots, D_{K}(\mathbf{A})\right\}^{T}$. Since it is rarely the case that a single set of decision variables simultaneously optimizes all the objective functions, "trade-offs" between multiple solutions for each objective function are sought. The notion of "optimum" is consequently redefined as Pareto optimum. A particular vector of decision variables $\mathbf{A}^{*} \in F$ is called Pareto optimal if there exists no feasible vector of decision variables such as $\mathbf{A} \in F$, which could decrease some criterion however without causing a simultaneous increase in at least one of the other criteria. Mathematically, this optimization rule can be expressed as follows: there does not exist another $\mathbf{A} \in F$ such that

$$
D_{k}(\mathbf{A}) \leq D_{k}\left(\mathbf{A}^{*}\right), \quad \forall k=1, \ldots, K .
$$

This rule rarely generates Pareto-optimal solutions and its plot is generally referred to as the Pareto front. The vectors $\mathbf{A}^{*}$ are usually called nondominated set.

PAES is a multiobjective local search method. It comprises three parts: the candidate solution generator, the candidate solution acceptance function, and the nondominated solutions (NDS) archive. The candidate solution generator is similar to random mutation hill-climbing. It maintains a single current solution and, in each iteration, produces a new candidate via random mutation. The design of the acceptance function is obvious in the case of the mutant dominating the current solution or vice versa, but in the nondominated case, a comparison set is used to help decide between the mutant and the current solution. Thus, an NDS list is needed to explicitly maintain a limited number of the nondominated solutions when they are found by the hill-climber, as the aim of multiobjective search is to find a spread of nondominated solutions. In [19], a pseudocode showing the simplest form of PAES was given in Algorithm 1.

A grid is used in PAES in order to ensure archived points cover a wide extent in objective space and are "well distributed." It is done by recursively dividing the $d$-dimensional objective space. For this, an adaptive grid archiving algorithm (AGA) can be used [14, 19]. When each solution is generated, its grid location in objective space is determined. Assuming the range of the space is defined in 


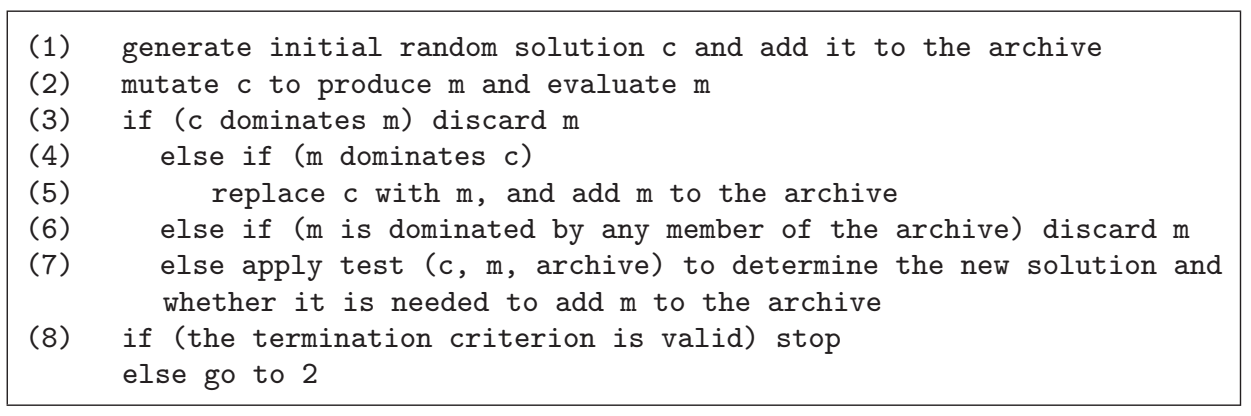

Algorithm 1: PAES algorithm.
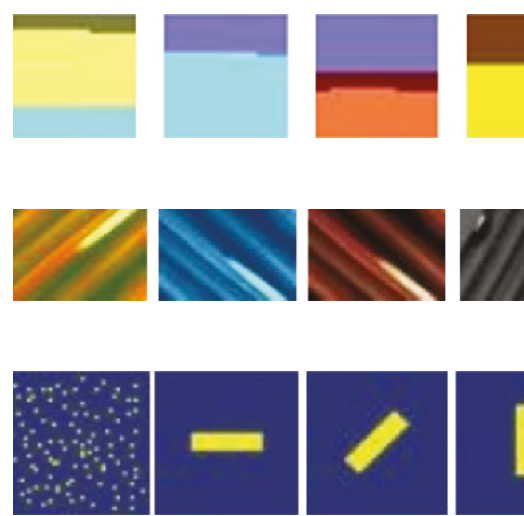
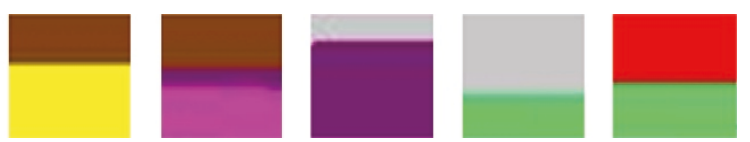

(a)
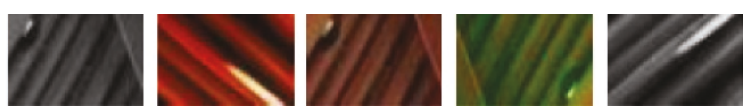

(b)
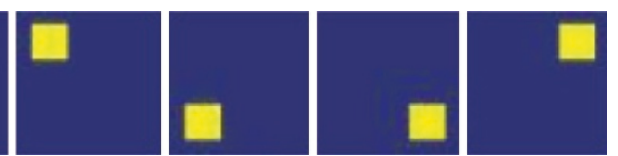

(c)

Figure 6: Three groups of 8 image blocks which well-defined low-level characteristics.

each objective, the required grid location can be found by repeatedly bisecting the range in each objective and finding the half where the solution lies. The recursive subdivision of the space and assignment of grid location is carried out in AGA by calculating the range in objective space of the current solutions in the archive and adjusting the grid so that it covers this range.

In the algorithm, the uniquely extremal vectors are protected from being removed from the archive once they have entered it (except by domination). Thus, vectors in the archive will converge to a set which covers the largest possible range in objective space in each objective. On the other hand, the archiving algorithm will be removing vectors from crowded regions. A comprehensive comparative study of several well-known algorithms for MOO was conducted in [20]. As a result, PAES appears as one of the best techniques showing very low complexity.

\subsection{Similarity matching in optimal multifeature space}

For a particular predefined concept, an optimal multifeature combination factor set $\mathbf{A}$ is obtained from the optimization step. Using this set of combination factors, the similarity distance for any block can be calculated by

$$
D(\mathbf{V}, \overline{\mathbf{V}}, \mathbf{A})=\sum_{l=1}^{L} \alpha_{l} d_{l}\left(\mathbf{v}_{l}, \overline{\mathbf{v}}_{l}\right)
$$

These distance estimations are supposed to be representing how likely an image block region contains a particular concept. Using these distances alone, a complete image retrieval process can be achieved without following steps of the work. In this approach, the mapping from block level to image level is achieved by using the similarity of the most similar block of an image to the concept as the similarity of the image to the concept.

\section{EXPERIMENTS}

As mentioned in Section 2, seven image low-level primitives were used to assess the performance of the proposed approach: CLD, CSD, DCD, EHD [6], TGF [15], GLC [16], and HSV [17]. It is important to stress that the proposed approach is not tailored to a given number of low-level descriptors. Any descriptor bank can be used.

The first set of experiments used selected blocks from synthetic and natural images. It aimed at showing the effectiveness of the weights derived from images with very obvious similarity in a given feature space but large differences in others. The goal was to validate the effectives of the proposed technique in well-defined scenarios. Initially, the "hori" set shown in Figure 3 was considered. It was obvious that the edge descriptor clearly dominates other features. Thus, classification based on an edge feature would outperform the same classifier using any other feature. When using the MOO-based approach, a weight of 0.9997 was derived for 
TABLe 1: Weights obtained for the three groups of blocks depicted in Figure 6 using different descriptors.

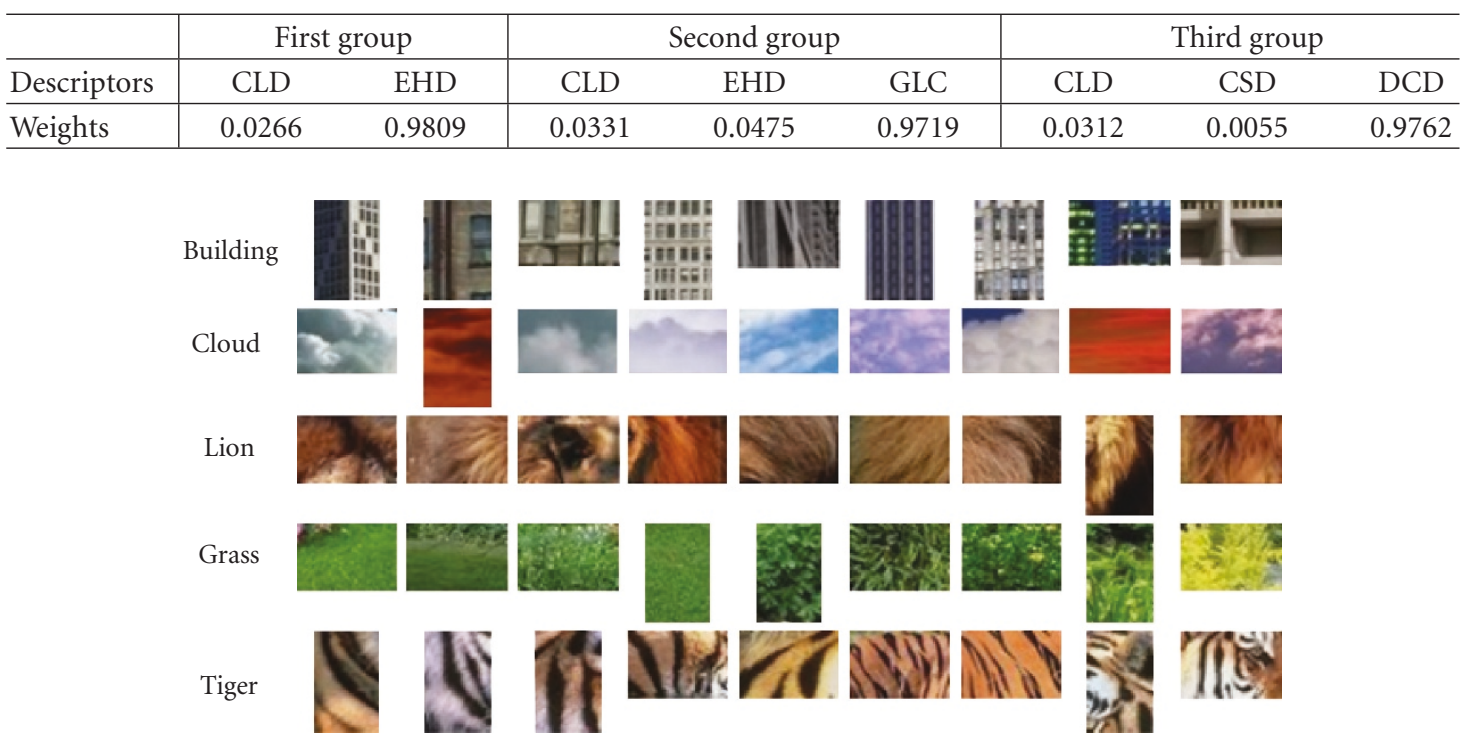

FIGURE 7: Samples of representative blocks considered for concepts: building, cloud, lion, grass, and tiger.

and assigned to the edge descriptor. Clearly, all other descriptors were ignored. It could be concluded that using $\mathrm{MOO}$ to find the optimal metric in multidescriptor space for image classification safely outperformed techniques using the "best" single descriptors. To consolidate this early conclusion, additional experiments were run. Figure 6 shows three groups of 8 image blocks each.

The first group at the top row in Figure 6 featured clear horizontal edges and a diversity of colors. The CLD and EHD descriptors were tested on this group. The weights for each descriptor as estimated by the MOO approach were shown in Table 1. The second group of blocks featured similar texture and a variety of colors and edge orientations. The CLD, EHD, and GLC descriptors were combined in this case. The weights for each descriptor as returned by the MOO approach were shown in Table 1. Experiments on the next group aimed at showing that even for different descriptors based on the same visual cue (color), the proposed approach showed a good discriminative power. The third group showed a group of 8 blocks consisting of exactly the same set of pixels: 100 yellow and 1500 blue pixels. However, the distribution and arrangement of the pixels were different in each block. The weights for each descriptor returned by the proposed approach were shown in Table 1. Clearly, the DCD should dominate in this case while the CLD and CSD present a clear coefficient variation across the group.

From these experiments it could be observed that the proposed approach assigned suitable weights to each feature space according to the low-level characteristics of the date set. This set of experiments also showed that for images with similar low-level characteristics, the best descriptor was selected from the descriptor bank and the approach reduced to a single objective optimization.

In the second round of experiments a small dataset containing 700 natural images with known ground truths were considered. This annotated set was created using natural pictures from the Corel database. All images were manually selected and labelled according to five predefined concepts: building, cloud, lion, grass, and tiger. The images representing these five different semantic concepts were then mixed to create the dataset.

Since ground truths were available in this case, precision and recall of the retrieval performance were estimated. Four groups of 20 elementary representative blocks were manually selected to represent each concept: 10 positive and 10 negative samples. For each group, the distance matrix (3) was defined using these 20 blocks and the 7 descriptors mentioned at the beginning of this section: CLD, CSD, DCD, EHD, TGF, GLC and HSV [6, 15-17]. Thus, 20 multiobjective functions of 7 variables were defined. Some of the sample blocks for the different concepts were depicted in Figure 7. The set of weights obtained after 10000 iterations of the PAES algorithm were shown in Table 2. To guarantee a good performance of the metric build with the coefficients returned by PAES, while keeping the computation cost, 10000 iterations were considered as a good (empirically estimated) trade-off.

Using these weights, the similarity between each block in the dataset and the virtual centroid of a concept was estimated. A similarity ranking list of blocks was then generated. If a block of an image was relevant to a concept, the image itself was considered relevant to that concept. Using this rule, images were ranked according to the ranking of single blocks. Finally, the precision-recall curves for each concept were estimated. Precision and recall values were used as the major evaluation criteria in this paper. They are commonly defined as follows:

$$
\begin{gathered}
\text { precision }=\frac{\text { retrieved and relevant }}{\text { retrieved }}, \\
\text { recall }=\frac{\text { retrieved and relevant }}{\text { relevant }} .
\end{gathered}
$$


TABLE 2: Weights obtained after 10000 iterations of PAES.

\begin{tabular}{l|ccccccc}
\hline & CLD & CSD & DCD & EHD & TGF & GLC & HSV \\
\hline Building & 0.6736 & 0.0537 & 0.0095 & 0.0353 & 0.0804 & 0.0858 & 0.0646 \\
Cloud & 0.0252 & 0.7780 & 0.0914 & 0.0761 & 0.1833 & 0.0293 & 0.0315 \\
Grass & 0.130 & 0.1941 & 0.1526 & 0.3296 & 0.0460 & 0.1830 & 0.0039 \\
Lion & 0.196 & 0.4684 & 0.0677 & 0.0599 & 0.1176 & 0.0516 & 0.0415 \\
Tiger & 0.0380 & 0.0604 & 0.0040 & 0.3474 & 0.3004 & 0.1689 & 0.0895 \\
\hline
\end{tabular}

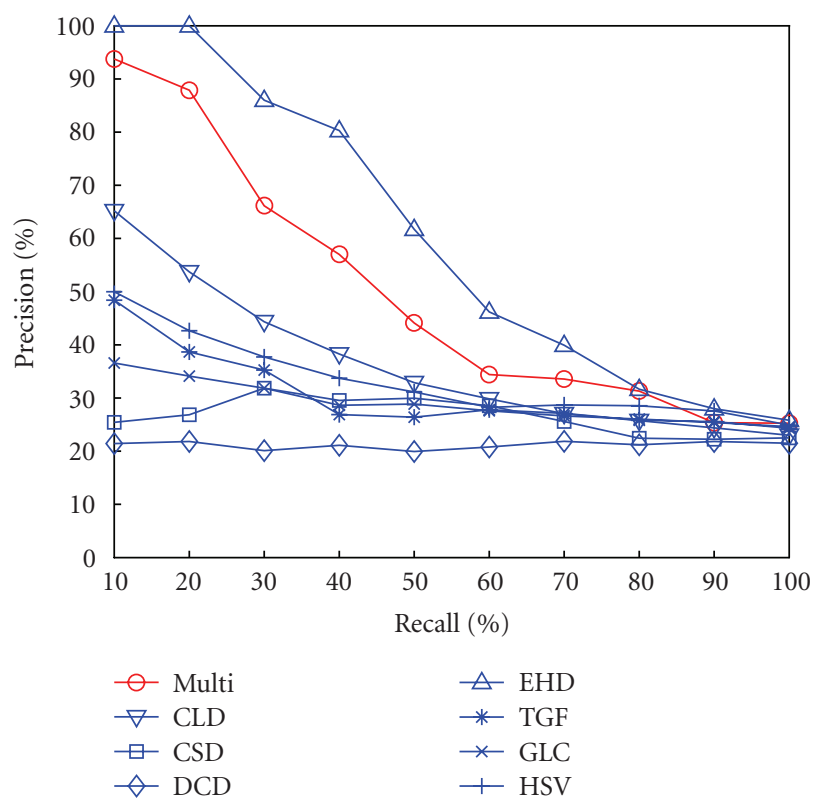

FIgURE 8: Precision-recall curves for concept building using the multifeature combination metric and using single descriptors.

In order to prove that the multifeature combination metric performs better than any of the combined single descriptors, the precision-recall curves using single descriptors were also plotted in the same diagrams for comparison. These curves were depicted in Figures 8-12, each of which plotted curves for one of the 5 predefined concepts.

In Figures 8-12, the curves obtained using our proposed approach was plotted with mark "o," and they were labelled as "multi" since the major outcome of our method was to find out the optimised multifeature space. The curves obtained using each of the single descriptors were plotted with other marks.

It could be observed from Figures 8-12 that the retrieval performance using the proposed approach was only slightly outperformed in a single case: for building using EHD, but the disadvantage of the proposed approach is not big. This is due to the prevailing dominance of EHD for building pictures. However, EHD cannot be prevailing dominant for any concept, and there is no such a "super descriptor" can really do so. This is why an approach such as the proposed one is needed, which approximates the "super descriptor" by combining several properly selected descriptors. It is not required that this approximated "super descriptor" outperforms any single descriptor when searching for any concept. Rather, the

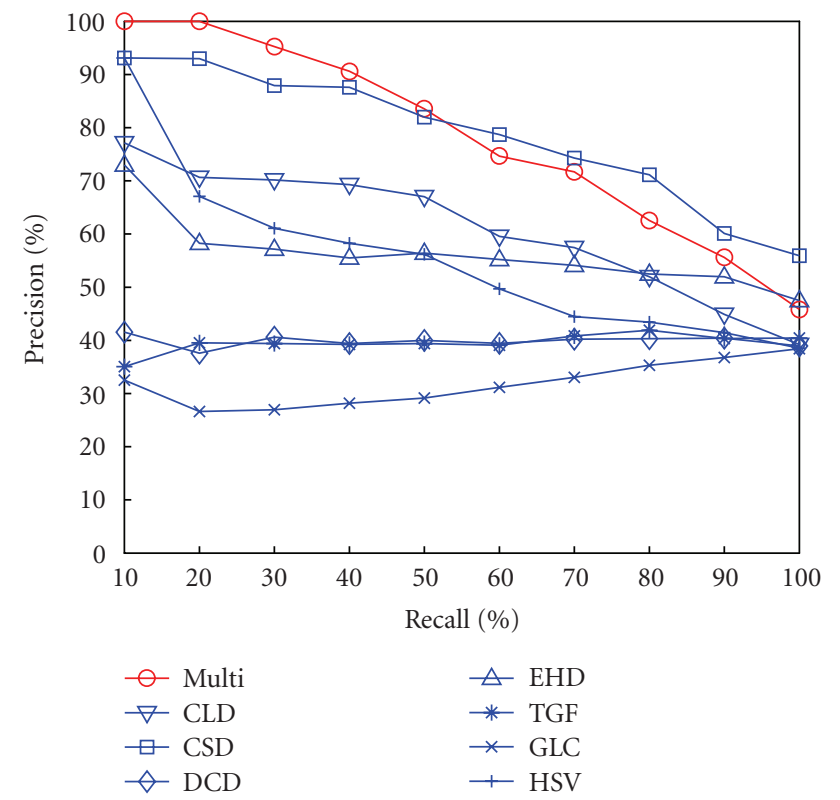

FIGURE 9: Precision-recall curves for concept cloud using the multifeature combination metric and using single descriptors.

aim is to have it perform not worse than, if not better than, any single descriptor when searching for any concept. Therefore, the result given in Figure 8 is acceptable given the aim of the proposed approach. In other cases shown in Figures 912 , the proposed approach performed better than using any single descriptor in the sense of both precision and recall.

When the multifeature space was applied in a complete image retrieval system, usually the results were displayed in a graphical user interface. Retrieved images were presented to the user in a ranking order according to their visual similarity. Here, a threshold was needed to define how many most similar images were to be displayed. In our framework, this threshold value was modifiable, but it was set to be 50 by default. The precision value was redefined as follows:

$$
\text { precision }=\frac{\text { retrieved-by-threshold and relevant }}{\text { retrieved-by-threshold }} \text {. }
$$

The precision values on the first displaying page with a threshold value of 50 were presented in Table 3 . In the literature, many other approaches of multifeature fusion had been proposed [8-12]. However, some of these approaches were very different from ours in the sense of comparability [9-11]. Some others employed human interactions and were based on different test datasets, so restoring their environment for 
TABLe 3: Precision values for the first page of retrieved images with a threshold of 50.

\begin{tabular}{|c|c|c|c|c|c|c|c|c|}
\hline & Multifeature metric & CLD & $\mathrm{CSC}$ & $\mathrm{DCD}$ & EHD & TGF & GLC & HSV \\
\hline Building & $\mathbf{9 0} \%$ & $65.00 \%$ & $30.00 \%$ & $20.00 \%$ & $90.00 \%$ & $50.00 \%$ & $25.00 \%$ & $55.00 \%$ \\
\hline Cloud & $95 \%$ & $75.00 \%$ & $95.00 \%$ & $40.00 \%$ & $75.00 \%$ & $25.00 \%$ & $35.00 \%$ & $100.00 \%$ \\
\hline Grass & $100 \%$ & $95.00 \%$ & $95.00 \%$ & $30.00 \%$ & $95.00 \%$ & $65.00 \%$ & $90.00 \%$ & $95.00 \%$ \\
\hline Lion & $85 \%$ & $65.00 \%$ & $55.00 \%$ & $20.00 \%$ & $65.00 \%$ & $25.00 \%$ & $45.00 \%$ & $75.00 \%$ \\
\hline Tiger & $70 \%$ & $5.00 \%$ & $55.00 \%$ & $5.00 \%$ & $25.00 \%$ & $30.00 \%$ & $25.00 \%$ & $65.00 \%$ \\
\hline
\end{tabular}

TABLE 4: Our results comparing with precision values at the second iteration, presented in [12].

\begin{tabular}{|c|c|c|c|c|}
\hline & Proposed multifeature metric & LK (CONC) in [12] & $\mathrm{RBF}(\mathrm{CONC})$ in [12] & ACK in [12] \\
\hline Building & $\mathbf{9 0} \%$ & $62 \%$ & $73 \%$ & $75 \%$ \\
\hline Cloud & $95 \%$ & $61 \%$ & $73 \%$ & $92 \%$ \\
\hline Grass & $100 \%$ & $29 \%$ & $28 \%$ & $42 \%$ \\
\hline Lion & $\mathbf{8 5} \%$ & $37 \%$ & $46 \%$ & $59 \%$ \\
\hline Tiger & $70 \%$ & $64 \%$ & $49 \%$ & $60 \%$ \\
\hline
\end{tabular}

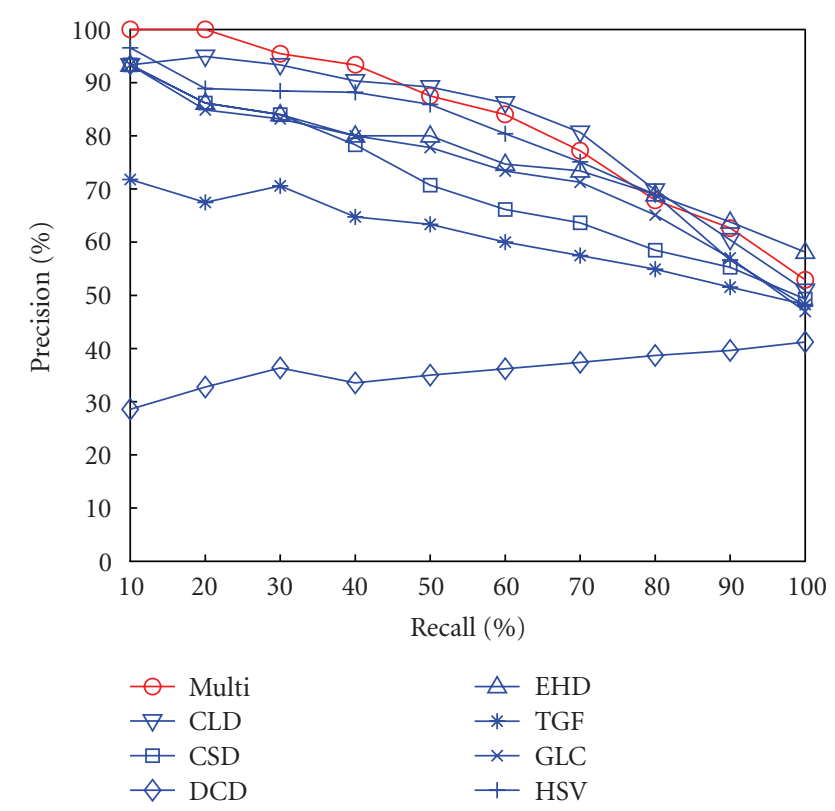

FIgURE 10: Precision-recall curves for concept grass using the multifeature combination metric and using single descriptors.

comparison was almost infeasible [8]. Since in [12], a set of experiments used the same dataset as we used here, their results were taken as a comparison with our approach. These results were presented in Table 4 . In [12], the authors presented approaches employing several different combinations of low-level features and SVM kernels. Among these kernels, the approaches using RBF kernel, Laplace kernel, and the adaptive convolution kernel (ACK) for combining the same 7 visual descriptors generally performed best. Moreover, the method in [12] was based on user relevance feedback. The precisions were generally the highest at the second iteration of relevance feedback. For the sake of comparability, the re-

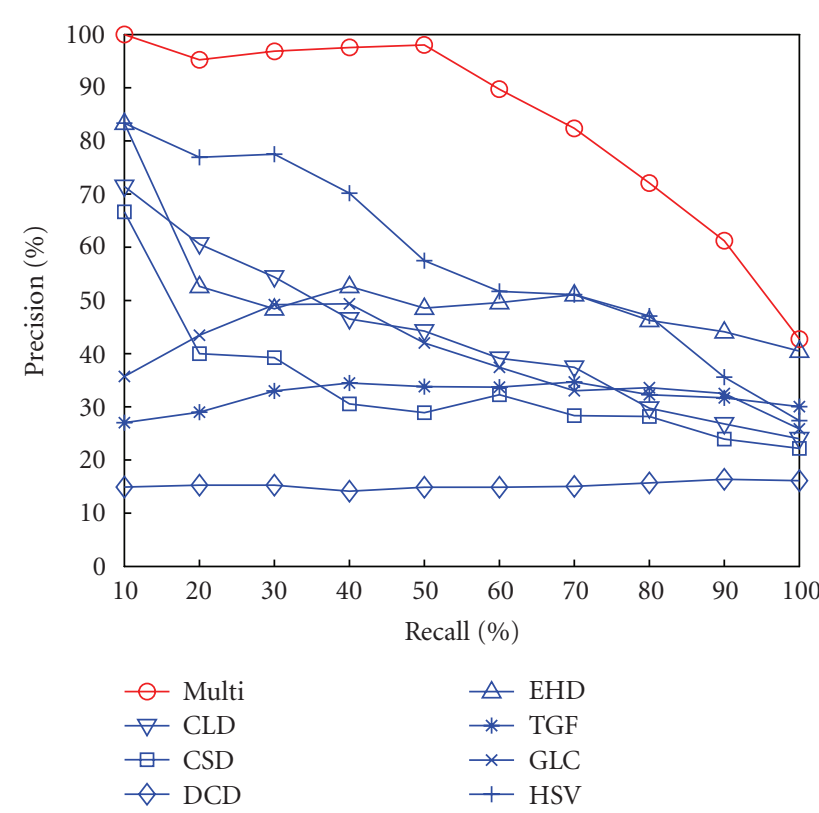

FIGURE 11: Precision-recall curves for concept lion using the multifeature combination metric and using single descriptors.

sults obtained using the above three kernels at the second iteration were chosen and presented.

As shown in Table 3, retrievals using the proposed multifeature metric generally outperformed retrievals using any of the single descriptors in the perspective of precision. Comparing with the approach proposed in [12], our approach was relatively more accurate. Besides, results listed in Table 4 were obtained after 2 iterations of relevance feedback, while our approach was fully automatic.

The next set of experiments used a more realistic (larger) dataset containing 12700 images from the Corel database. Experiments based on this dataset aimed at validating the 


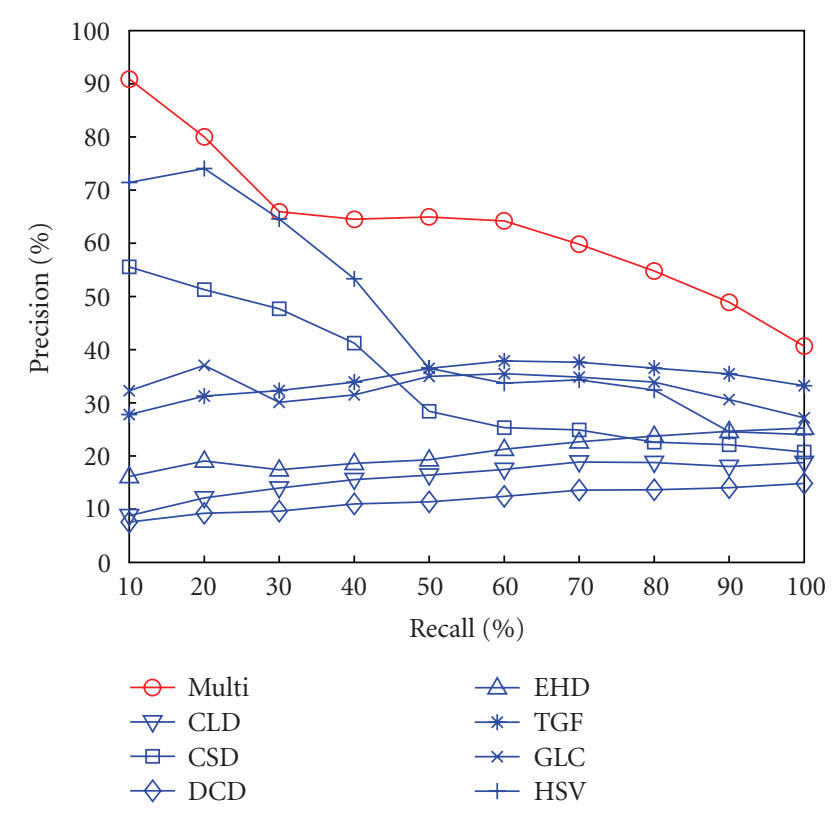

FIgURE 12: Precision-recall curves for concept tiger using the multifeature combination metric and using single descriptors.

proposed approach within a realistic natural image retrieval scenario. The goal of our approach in such scenarios was to effectively gather the relevant images at the beginning of image ranking list, so that users could find a dense collection of images on their demand in the retrieved images.

This dataset contained a large number of images and many kinds of objects including animals, plants, human, sceneries, and events. Even for the most common concepts like grass, the images containing each concept were sparsely spread over in the whole set. Because no ground truth was available here due to the dataset size, only the first several hundreds retrieved images were judged as relevant or irrelevant in a subjective fashion by a human evaluator. The first 500 retrieved images were manually judged for performance evaluation. Starting from 10, when every 10 more images were retrieved, a precision value was estimated as in (13). To compensate for the sparseness of the relevant images for each concept, the obtained precision values were compared with the percentages of each concept in the dataset. These percentages were manually calculated from a random subset of 1000 images in the whole dataset.

Figure 13 showed the precisions curves against the number of retrieved images for a few concepts. The straight lines in Figure 13 indicated the percentages of images in the dataset for the corresponding concept, that is, curves and lines with the same colors and marks represented the same concepts. However, these curves were not the complete precision curves for the concepts. They were just very small segments at the beginning of the complete curves, that is, the segments of first 500/12700 of the complete curves.

Figure 14 showed the retrieval recall curves against the number of retrieved images for concepts lion and tiger. Recall values were estimated as in (12) based on the known fact that there were 100 images containing each of these two concepts

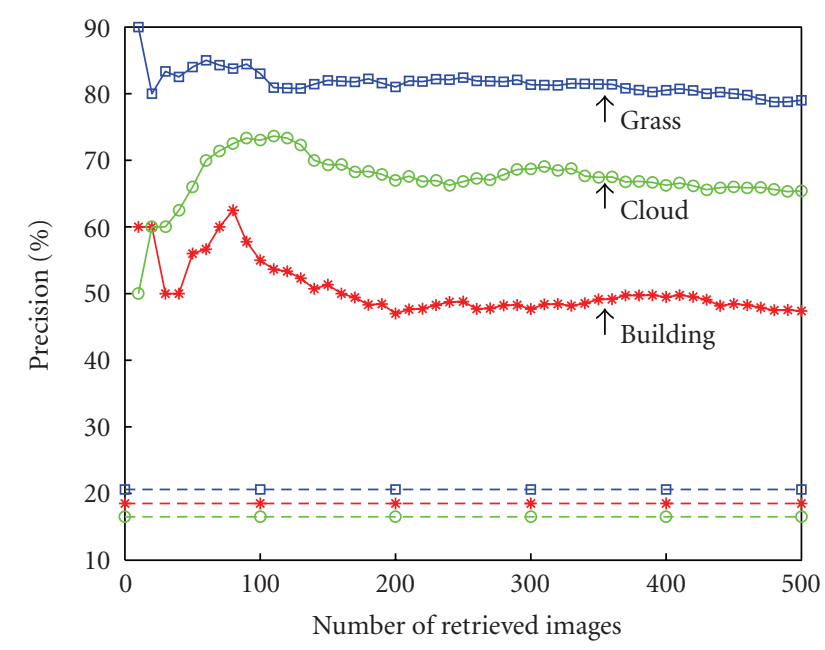

FIGURE 13: The retrieval precisions curves for 3 concepts compared with the average percentages.

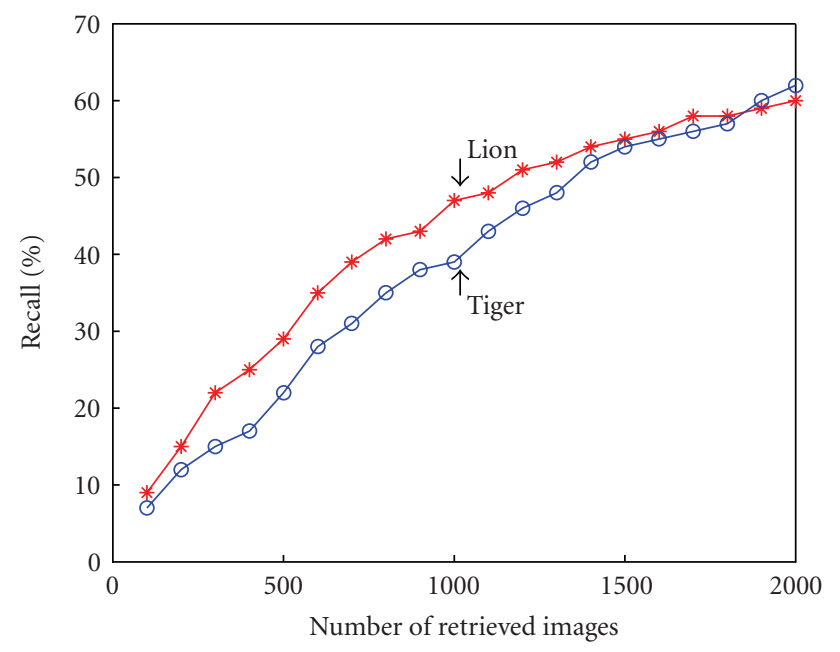

Figure 14: The retrieval recall curves of 2 concepts.

in the dataset. Same as experiments presented in Figure 13, no ground truth was available for these experiments. Therefore, only 2000 images were considered as a good representation since there were only over 100 images for each concept in the database. However similarly, these curves were not the complete recall curves for the concepts. They were just very small segments at the beginning of the complete curves, that is, the segments of first 2000/12700 of the complete curves.

It could be observed from Figures 13 and 14 that relevant images for each concept were effectively gathered within the first few hundreds in the ranking list. It can be concluded that the proposed approach has good discriminative power and it is suitable for retrieving natural images in large datasets.

\section{CONCLUSIONS}

An approach for semantic-based and object-oriented image retrieval is presented. By analysing the visual content of a group of representative image blocks, an optimal similarity 
metric per semantic concept is obtained. The core of the proposed approach is a multiobjective optimization technique to estimate weights for a linear combination of single metrics in multifeature space. The proposed approach has been tested by retrieving natural images in representative datasets. A comprehensive evaluation of the proposed technique is presented. Based on the evaluation, we proved the ability of the proposed approach to effectively retrieve images according to semantic concepts from large natural image databases.

\section{ACKNOWLEDGMENT}

This research was partially supported by the European Commission under the Contract FP6-045189, RUSHES.

\section{REFERENCES}

[1] C. Bishop and C. M. Bishop, Neural Networks for Pattern Recognition, Oxford University Press, Oxford, Miss, USA, 1995.

[2] O. Chapelle, P. Haffner, and V. N. Vapnik, "Support vector machines for histogram-based image classification," IEEE Transactions on Neural Networks, vol. 10, no. 5, pp. 1055-1064, 1999.

[3] N. Friedman, D. Geiger, and M. Goldszmidt, "Bayesian network classifiers," Machine Learning, vol. 29, no. 2, pp. 131-163, 1997.

[4] A. W. M. Smeulders, M. Worring, S. Santini, A. Gupta, and R. Jain, "Content-based image retrieval at the end of the early years," IEEE Transactions on Pattern Analysis and Machine Intelligence, vol. 22, no. 12, pp. 1349-1380, 2000.

[5] Y. Rui, T. S. Huang, and S.-F. Chang, "Image retrieval: current techniques, promising directions, and open issues," Journal of Visual Communication and Image Representation, vol. 10, no. 1, pp. 39-62, 1999.

[6] S.-F. Chang, T. Sikora, and A. Purl, "Overview of the MPEG-7 standard," IEEE Transactions on Circuits and Systems for Video Technology, vol. 11, no. 6, pp. 688-695, 2001.

[7] A. Mojsilović, "A computational model for color naming and describing color composition of images," IEEE Transactions on Image Processing, vol. 14, no. 5, pp. 690-699, 2005.

[8] Y. Rui, T. S. Huang, M. Ortega, and S. Mehrotra, "Relevance feedback: a power tool for interactive content-based image retrieval," IEEE Transactions on Circuits and Systems for Video Technology, vol. 8, no. 5, pp. 644-655, 1998.

[9] S. Newsam, B. Sumengen, and B. S. Manjunath, "Categorybased image retrieval," in Proceedings of IEEE International Conference on Image Processing (ICIP '01), vol. 3, pp. 596-599, Thessaloniki, Greece, October 2001.

[10] C. Schmid and R. Mohr, "Local grayvalue invariants for image retrieval," IEEE Transactions on Pattern Analysis and Machine Intelligence, vol. 19, no. 5, pp. 530-535, 1997.

[11] M. Soysal and A. A. Alatan, "Combining MPEG-7 based visual experts for reaching semantics," in Proceedings of the 8th International Workshop on Visual Content Processing and Representation (VLBV'03), vol. 2849 of Lecture Notes in Computer Science, pp. 66-75, Madrid, Spain, September 2003.

[12] D. Djordjevic and E. Izquierdo, "An object- and user-driven system for semantic-based image annotation and retrieval," IEEE Transactions on Circuits and Systems for Video Technology, vol. 17, no. 3, pp. 313-323, 2007.

[13] R. E. Steuer, Multiple Criteria Optimization: Theory, Computation, and Application, John Wiley \& Sons, New York, NY, USA, 1986.
[14] J. Knowles and D. Corne, "Approximating the nondominated front using the pareto archived evolution strategy," Evolutionary Computation, vol. 8, no. 2, pp. 149-172, 2000.

[15] B. S. Manjunath and W. Y. Ma, "Texture features for browsing and retrieval of image data," IEEE Transactions on Pattern Analysis and Machine Intelligence, vol. 18, no. 8, pp. 837-842, 1996.

[16] M. Tuceryan and A. K. Jain, "Texture analysis," in The Handbook of Pattern Recognition and Computer Vision, pp. 207-248, World Scientific, River Edge, NJ, USA, 2nd edition, 1998.

[17] M. J. Swain and D. H. Ballard, "Color indexing," International Journal of Computer Vision, vol. 7, no. 1, pp. 11-32, 1991.

[18] Q. Zhang and E. Izquierdo, "A multi-feature optimization approach to object-based image classification," in Proceedings the 5th International Conference on Image and Video Retrieval (CIVR '06), vol. 4071 of Lecture Notes in Computer Science, pp. 310-319, Tempe, Ariz, USA, July 2006.

[19] J. Knowles and D. Corne, "Properties of an adaptive archiving algorithm for storing nondominated vectors," IEEE Transactions on Evolutionary Computation, vol. 7, no. 2, pp. 100-116, 2003.

[20] A. Jaszkiewicz, Multiple Objective Metaheuristic Algorithms for Combinatorial Optimization, Poznan University of Technology, Poznan, Poland, 2001. 